Proceedings of the 46th International School and Conference on the Physics of Semiconductors "Jaszowiec" 2017, Szczyrk

\title{
In Situ Raman Spectroscopy of Solution-Gated Graphene on Copper
}

\author{
J. BindeR ${ }^{a, *}$, R. StęPnieWski ${ }^{a}$, W. Strupiński ${ }^{b}$ And A. WysmoŁeK ${ }^{a}$ \\ ${ }^{a}$ Faculty of Physics, University of Warsaw, L. Pasteura 5, 02-093 Warsaw, Poland \\ ${ }^{b}$ Institute of Electronic Materials Technology, Wólczyńska 133, 01-919 Warsaw, Poland
}

\begin{abstract}
We present a solution-gated in situ Raman spectroscopy approach, which enables the electrical characterization of graphene on a copper substrate without the need of a transfer process. The application of a voltage across the solution resulted in a shift of the Raman $G$-band without a significant shift of the $2 D$ band. This observation allowed for the separation of the effects of strain and doping. Based on the $G$ and $2 D$ band shifts we show that we can manipulate the $n$-type carrier concentration of graphene directly on the copper substrate in a range from about $8 \times 10^{12} \mathrm{~cm}^{-2}$ to about $1.5 \times 10^{13} \mathrm{~cm}^{-2}$.

DOI: 10.12693/APhysPolA.132.360

PACS/topics: 68.65.Pq, 78.67.Wj, 73.30.+y
\end{abstract}

\section{Introduction}

The physics of graphene has been explored in great detail over the last decade [1]. Many breakthroughs and exceptional findings in fundamental physics were reported, which resulted in a profound knowledge of the physical effects as well as the development of powerful characterization methods for graphene [2]. Different device themes were also proposed and their feasibility was shown mostly in the form of proof of principle prototypes in the laboratories [3]. Despite this tremendous progress, the application of graphene for real-world problems is still lagging behind. A major bottleneck for industrial graphene device implementation is the lack of a high-quality, low-cost method to derive graphene. All of the methods reported so far have their specific drawbacks and although new methods are constantly being developed [4], there is still room for innovation. In this report we would like to address the difficulties of the currently most commonly employed technique, which is graphene growth by chemical vapor deposition (CVD) on copper. This growth method readily allows for the growth of large area graphene. However, the spectrum of application for graphene on copper is very limited due to the conductivity of the metal substrate. Thus, in order to use such graphene for electrical applications it has to be transferred to an insulating substrate. It has been shown that the transfer process itself (e.g. by wet chemical etching of the metal foil) can strongly degrade the electrical properties of graphene. It would therefore be desirable to have the means to assess the properties of graphene directly on the metal foil without the impact of the transfer procedure [5].

In this work we report on a novel measurement approach that allows us to study the carrier concentration of

*corresponding author; e-mail: johannes.binder@fuw.edu.pl graphene directly on a metal foil. To this end we employ a combination of Raman spectroscopy and solution gating. It is well established that with Raman spectroscopy on graphene one can deduce the desired information by monitoring the shift of the $G$ band caused by an alternation of the carrier concentration [6, 7]. However, this method requires the possibility to change the carrier concentration, which on a highly conductive substrate cannot be performed using conventional solid dielectric based gating techniques and resistance measurements since the substrate always masks the changes induced in graphene.

\section{Experimental}

For the measurements presented in this report a dedicated apochromatic water-dipping objective with 40x magnification and a numerical aperture of 0.8 was used. This objective allows to focus the laser spot by immersing the objective directly into the aqueous solution $(0.1 \mathrm{M}$ $\mathrm{NaCl}$, ACS grade). The graphene on copper sample was placed inside a teflon cell with fluoroelastomer O-ring sealing. The electrical connections to the sample were made outside of the solution basin with silver paint. A high purity silver wire was used as counter electrode and for the electrical measurements a Keithley 2400 sourcemeter was employed. The Raman spectra were recorded using a Horiba T64000 spectrometer with a liquid nitrogen cooled CCD camera and a $633 \mathrm{~nm}$ He-Ne laser.

\section{Results and discussion}

For solution gated measurements it is very important to establish the voltage window for which one can neglect the impact of electrochemical reactions [7]. In contrast to previous studies on $\mathrm{SiC}$ [8], one is by far more limited in the case of a copper substrate due to a rich electrochemical spectrum in aqueous solutions [9]. In order to establish the limits for which one can assume that no electrochemical reactions take part, one has to closely monitor the current passing through the solution. 
For the region corresponding to Fig. 1, a nearly constant background current without any peak-like features was observed. Knowing the voltage range that we can apply, we can overcome the problem regarding the conductive substrate by taking advantage of the fact that the double layer that builds up at the graphene-solution interface is giving rise to a large electric field only in the vicinity of the interface. The changes induced by charged species accumulating in the solution are screened by the charge carriers in the graphene layer and the metal underneath. Since we are only interested in the surface (i.e. the graphene), we can separate the effects on carbon and on the metal with the use of Raman spectroscopy. This sensitivity to the surface is the big advantage over conventional electrical transport.

Figure 1 presents the results of a sweep in applied voltage and simultaneous Raman spectroscopy for the $G$ and $2 D$ band. Please note that a luminescence background has been subtracted for all the measurements shown.

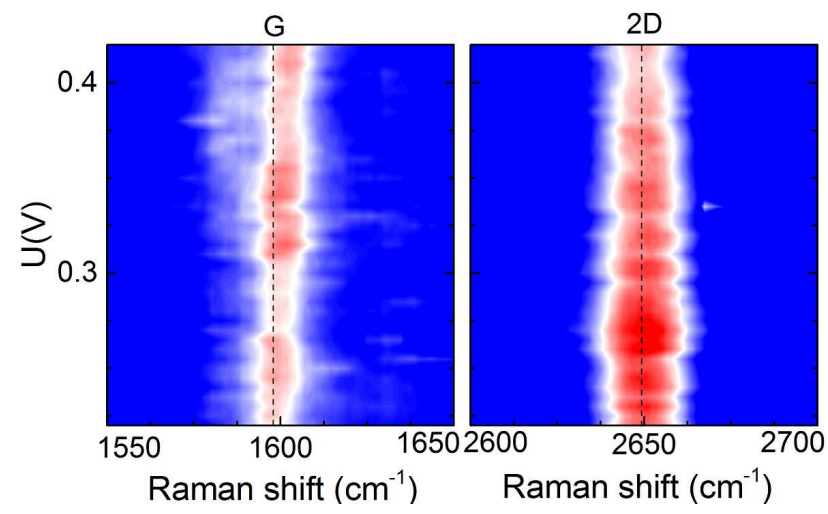

Fig. 1. Raman intensity false color maps of the $G$ band (left panel) and the $2 D$ band (right panel) Raman shift as a function of applied voltage to the solution gate.

For the false color plot one can clearly observe a blueshift of the $G$ band for larger voltages. The position of the $2 D$ band, however, remains largely unaffected by the applied voltage. In order to probe the reproducibility, a sweep in the opposite direction followed by another complete sequence of two sweeps in opposite directions was performed in one continuous measurement showing the same results, i.e. a blueshift of the $G$ band for larger voltages. We can therefore rule out the influence of a prolonged laser irradiation to be responsible for the observed effects, since this cannot account for the redshift when performing a sweep in opposite voltage direction. In order to show the shift more markedly, three spectra that show the $G$ and $2 D$ band as a function of applied voltage are presented in Fig. 2 .

To further analyze the behavior, a single Lorentzian function was fitted to the $G$ and $2 D$ band of the spectra, which allowed to extract the Raman shift for each applied voltage. The results of the fits are shown in Fig. 3, for two consecutive sweeps in opposite directions. Again,

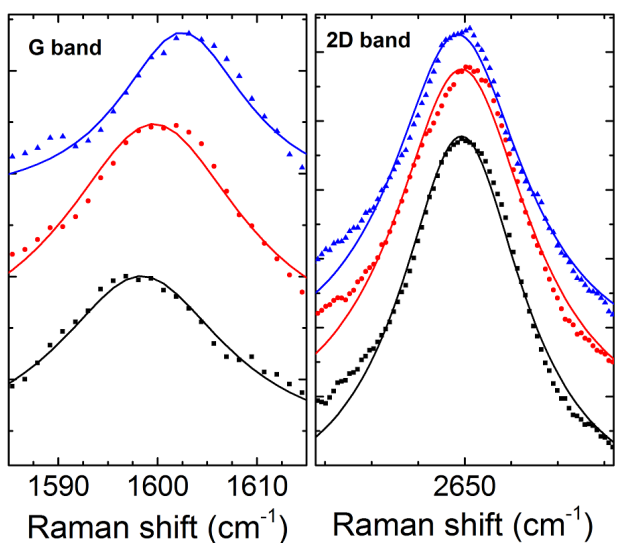

Fig. 2. Three spectra showing the $G$ and $2 D$ band for applied voltages of $0.22 \mathrm{~V}$ (black squares), $0.32 \mathrm{~V}$ (red circles) and $0.42 \mathrm{~V}$ (blue triangles) together with a single Lorentzian fit (solid line).

we clearly observe a shift for the $G$ band whereas the $2 D$ band is almost not affected. Only at voltages approaching $0.4 \mathrm{~V}$ a small redshift can be made out. By using this results we can estimate the carrier concentration and the changes induced by the solution gate. It has been shown that biaxial strain causes a movement of both the $G$ and $2 D$ band in the same direction [10]. In accordance with our expectations, changes in strain can therefore not explain a movement of the $G$ band only. Moreover, it has been shown that for moderate $n$-type doping the $2 D$ band position remains almost unaltered, whereas the $G$ band position moves a as a function of carrier concentration [6]. Thus, our measurements point towards $n$-type doping of graphene on copper immersed in solution. Literature data for graphene on copper not immersed in solution also report $n$-type doping [11]. With this information at hand, we can take the literature values for the $G$ and $2 D$ Raman shifts of unstrained, undoped graphene $\mathrm{E}_{G}=1580 \mathrm{~cm}^{-1}, E_{2 D 0}=2644 \mathrm{~cm}^{-1}[11-13]$ for a $633 \mathrm{~nm}$ laser excitation and estimate both strain and carrier concentration.

We measure a $2 D$ Raman shift of about $5 \mathrm{~cm}^{-1}$, which we can solely ascribe to effects of strain. The resulting strain should lead to a blueshift of the $G$ band of about $12 \mathrm{~cm}^{-1}[11,12]$. We hence expect a strained but undoped graphene to have a $G$ band Raman shift of $1592 \mathrm{~cm}^{-1}$. The lowest Raman shift we observe in our voltage range is at about $1598 \mathrm{~cm}^{-1}$, which means that the remaining $6 \mathrm{~cm}^{-1}$ come from $n$-type doping. We can estimate a concentration of about $8 \times 10^{12} \mathrm{~cm}^{-2}$, based on the assumption of a linear change in concentration of $1.35 \times 10^{12} \mathrm{~cm}^{-2}$ per $1 \mathrm{~cm}^{-1} G$ band blueshift $[6,12]$. This value is in good agreement with the range of concentrations observed in literature [11]. The changes induced by our gate lead to a further blueshift of about $5 \mathrm{~cm}^{-1}$, which corresponds to an additional change in concertation of about $7 \times 10^{12} \mathrm{~cm}^{-2}$. The maximum carrier concentration we obtained is hence of the order of 


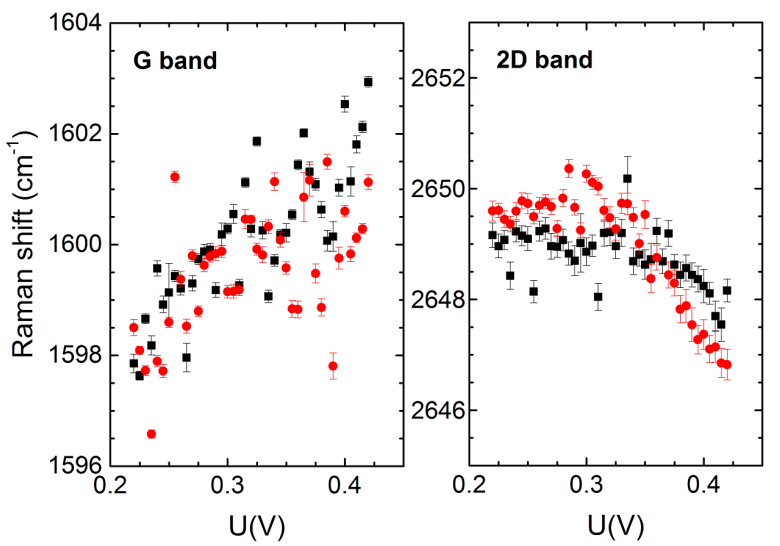

Fig. 3. Raman shift for the $G$ and $2 D$ band as a function of applied gate voltage extracted from single Lorentzian fits to the spectra. The black squares correspond to the measurement shown in Figures 1 and 2. The red circles show results for fits to the consecutive sweep in opposite voltage direction.

$1.5 \times 10^{13} \mathrm{~cm}^{-2}$. This carrier concentration falls into the upper limit for which one can assume the $2 D$ band to be nearly insensitive to changes in carrier concentration. Indeed, one expects the $2 D$ band to redshift for values exceeding this carrier concentration in excellent agreement to our observations [6].

\section{Conclusions}

With our approach we have shown a way to estimate material properties of graphene on copper like the carrier concentration by combined electro-optical measurements in aqueous solution. The specifics of the electrochemical gating, i.e. the build-up of a double layer, allows us to probe and manipulate changes in carrier concentration of the sample surface which in our case is the graphene layer. In-situ Raman spectroscopy was used to monitor the changes in doping, that manifests itself in a shift of the $G$ band. Taking together the behavior of the $G$ and $2 D$ band we could pinpoint the graphene layer to be $n$-type with an initial carrier concentration of about $8 \times 10^{12} \mathrm{~cm}^{-2}$. With the solution-gate we were able to alter the concentration to a level about $1.5 \times 10^{13} \mathrm{~cm}^{-2}$. We have shown that with the in-situ solution-gated Raman experiments one can evaluate and manipulate the doping of graphene even on metallic/conductive substrates. Hence, we obtain a useful tool to study the influence of the transfer process or, in combination with spatial mapping, the influence of grain boundaries on the local electrical properties of graphene on copper.

\section{Acknowledgments}

This work was supported by the National Science Centre, Poland grant 2014/13/N/ST3/03772.

\section{References}

[1] K.S. Novoselov, V. Fal'ko, L. Colombo, P. Gellert, M. Schwab, K. Kim, Nature 490, 192 (2012).

[2] A.C. Ferrari, D.M. Basko, Nat. Nanotechnol. 8, 235 (2013).

[3] R. Murali, in: Graphene Nanoelectronics, Springer, 2012 , p. 51.

[4] I. Pasternak, P. Dabrowski, P. Ciepielewski, V. Kolkovsky, Z. Klusek, J. Baranowski, W. Strupinski, $\mathrm{Na}$ noscale 8, 11241 (2016).

[5] R. Muńoz, C. Gómez-Aleixandre, Chem. Vap. Deposit. 19, 297 (2013).

[6] A. Das, S. Pisana, B. Chakraborty, S. Piscanec, S. Saha, U. Waghmare, K. Novoselov, H. Krishnamurthy, A. Geim, A. Ferrari, A. Sood, Nat. Nanotechnol. 3, 210 (2008).

[7] J. Binder, J. Urban, R. Stepniewski, W. Strupinski, A. Wysmolek, Nanotechnology 27, 045704 (2016).

[8] P.K. Ang, W. Chen, A.T.S. Wee, K.P. Loh, J. Am. Chem. Soc. 130, 14392 (2008).

[9] Y. Deng, A.D. Handoko, Y. Du, S. Xi, B.S. Yeo, ACS Catal. 6, 2473 (2016).

[10] T. Mohiuddin, A. Lombardo, R. Nair, A. Bonetti, G. Savini, R. Jalil, N. Bonini, D. Basko, C. Galiotis, N. Marzari, K.S. Novoselov, A.K. Geim, A.C. Ferrari, Phys. Rev. B 79, 205433 (2009).

[11] O. Frank, J. Vejpravova, V. Holy, L. Kavan, M. Kalbac, Carbon 68, 440 (2014).

[12] J. Urban, P. Dabrowski, J. Binder, M. Kopciuszynski, A. Wysmolek, Z. Klusek, M. Jalochowski, W. Strupinski, J. Baranowski, J. Appl. Phys. 115, 233504 (2014).

[13] D. Mafra, G. Samsonidze, L. Malard, D. Elias, J. Brant, F. Plentz, E. Alves, M. Pimenta, Phys. Rev. B. 76, 233407 (2007). 